\title{
Zolpidem-Induced Arousal by Paradoxical GABAergic Stimulation: A Case Report With F-18 Flumazenil Positron Emission Tomography and Single Photon Emission Computed Tomography Study
}

\author{
Changjae Kim, MD, Bum Sun Kwon, MD, Ki Yeun Nam, MD, \\ Jin Woo Park, MD, Ho Jun Lee, MD
}

Department of Physical Medicine and Rehabilitation, Dongguk University College of Medicine, Goyang, Korea

\begin{abstract}
Zolpidem is a non-benzodiazepine drug that has selectivity for the gamma-aminobutyric acid (GABA) receptors. We experienced paradoxical effect of zolpidem in a 48-year-old male patient with hypoxic-ischemic brain injury after cardiac arrest. The patient was in stupor and could not communicate. His Glasgow Coma Scale (GCS) was E2M4V2 and Rancho Los Amigos (RLA) was grade III to IV. Zolpidem was prescribed to induce sedation but paradoxically, he became alert (GCS 15, RLA VII) and was able to communicate. The arousal lasted for 2 hours repeatedly following each administration of the medication. While he was alert, electroencephalogram showed the reversal of slow wave into beta range fast activity and F-18 flumazenil positron emission tomography (PET) showed increased GABAergic receptor activity in both frontoparietotemporal cortices. Single photon emission computed tomography (SPECT) also showed increased cerebral perfusion and reversal of cerebellar diaschisis.
\end{abstract}

Keywords Hypoxia, Zolpidem, Arousal

\section{INTRODUCTION}

Zolpidem is a non-benzodiazepine drug that has selec-

Received June 1, 2015; Accepted July 29, 2015

Corresponding author: Bum Sun Kwon

Department of Physical Medicine and Rehabilitation, Dongguk University College of Medicine, 27 Dongguk-ro, Ilsandong-gu, Goyang 10326, Korea

Tel: +82-31-961-7460, Fax: +82-31-961-7488, E-mail: bskwon@dumc.or.kr

(c) This is an open-access article distributed under the terms of the Creative Commons Attribution Non-Commercial License (http://creativecommons. org/licenses/by-nc/4.0) which permits unrestricted noncommercial use, distribution, and reproduction in any medium, provided the original work is properly cited.

Copyright $\odot 2016$ by Korean Academy of Rehabilitation Medicine tivity for stimulating the effect of gamma-aminobutyric acid (GABA) and predominant affinity to the benzodiazepine receptor subtype omega-1 modulating $\mathrm{GABA}_{\mathrm{A}^{-}}$ benzodiazepine-chloride channel receptor complex, which mainly mediates sedation [1].

The first paradoxical zolpidem-induced arousal effect was reported in 2000, in a patient who had been in a semi-comatose state after a traffic accident; and several cases have been reported thereafter [1,2].

Subsequently, large prospective studies were conducted to prove the effect of zolpidem on vegetative state patients [3-5]. Improvement of cerebral state monitor and 
single photon emission computed tomography (SPECT) were shown after zolpidem administration [6]. However, only a small fraction of patients (4.8\%) showed improvement in cognition and behavior [3]. Furthermore, in one study, none of 60 subjects showed clinically significant improvement [4].

We reported a paradoxical effect of zolpidem that awakened stuporous mentality in a hypoxic-ischemic (HI) brain injured patient with brain imaging and electroencephalogram (EEG).

\section{CASE REPORT}

We experienced a 48-year-old male patient who had been bedridden and semi-comatose for 2 years after HI brain injury due to myocardial infarction. His Glasgow Coma Scale (GCS) was E2M4V2. He could respond to pain by eye opening and withdrawing and made several incomprehensible sounds. There were some spontaneous movements of both arms and hands, which were not purposeful. He showed agitated behaviors, which fluctuated and worsened at night. Rancho Los Amigos (RLA) score was grade III to IV. He was totally dependent in activities of daily living (ADL) and unable to ambulate. Thus, his Modified Barthel Index (MBI) was 0 and MiniMental Status Examination (MMSE) was 0.

Due to agitated behavior at night, we administered zolpidem (Stilnox $10 \mathrm{mg}$ ) 1 tablet for sleep induction. Thirty minutes after zolpidem administration, the patient became alert enough to talk and communicate with his wife. He responded appropriately to various stimuli and verbal commands. After administration of zolpidem, GCS was 15 (E4M6V5) and RLA score was VII. This arousal lasted for 2 to 3 hours and repeated every time of administration. Regular administration of zolpidem before meals made direct oral feeding possible instead of the use of percutaneous endoscopic gastrostomy tube. This improvement of swallowing without aspiration was confirmed by videofluoroscopic swallowing study. After administration of zolpidem, MMSE score improved from 0 to 12 , in which he responded appropriately in items of orientation, short-term memory, repetition and comprehension. MBI score improved from 0 to 13 . His motor performance showed improvement in carrying small objects in box and block test, and he was able to follow some simulated ADL, such as personal hygiene, dressing, bladder and bowel care with the therapists' guidance. He could walk with therapist's assistance and ambulate by wheelchair.

We attempted higher dosage of zolpidem (20 mg or 30 $\mathrm{mg}$ ), however, the patient fell asleep instead of showing arousal. Similarly, on administration of other GABAergic drugs and sleeping pills, he was sedated and fell asleep, which is the normal phenomenon in ordinary people.

Effect of the zolpidem on brain function was evaluated by EEG, brain PET and SPECT. Slow waves were recorded on EEG before medication (zolpidem-off), and the reversal of slow wave to beta range fast activity, which is dominant waveform in alert and attentive individuals, was detected after medication (zolpidem-on) (Fig. 1). In brain imaging, F-18 flumazenil positron emission tomography $\left(\left[{ }^{18} \mathrm{~F}\right]\right.$-FMZ-PET) showed increased GABA receptor activity in both frontoparietotemporal cortices at zolpidem-on, as compared to zolpidem-off (Fig. 2). SPECT also showed improvement of suppressed brain perfusion when zolpidem was administered (Fig. 3). Increased frequency of zolpidem administration to thrice daily, resulted in sustained improvement of alertness and functions throughout most of the daytime. After 1 month of regular administration of zolpidem, the cognitive and daily functions sustained (MMSE 13, MBI 13). The patient was discharged on a 10-mg of zolpidem thrice daily regimen. The effect of zolpidem lasted for more than 3 years.

\section{DISCUSSION}

This case report demonstrated that the stuporous patient became alert by administration of zolpidem. Likewise, Clauss and Nel [1] reported 3 patients with $\mathrm{HI}$ injury, who were in permanent vegetative state and aroused transiently after administration of zolpidem. While these case studies demonstrated increased consciousness level and cognitive function, we additionally experienced the improvement of functional activities, which was not evaluated by objective examination in previous studies. Brefel-Courbon et al. [5] also reported a similar case, in which zolpidem administration improved motor performance and neuropsychological status. They described the changes of brain metabolism by PET study and insisted that the activation of limbic loops is a possible motivational process. In our case, improvements in brain imaging included increased perfusion of left parietal 
(A)

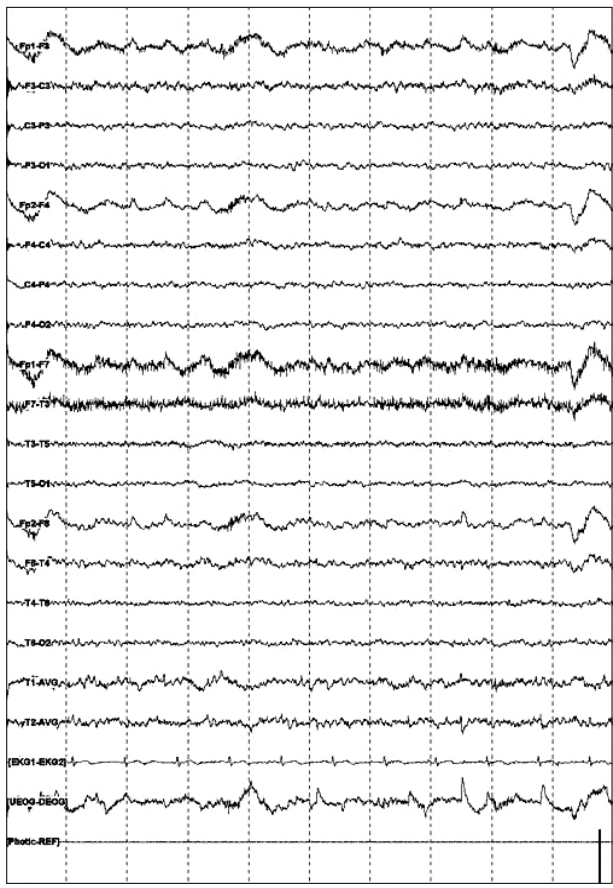

(B)

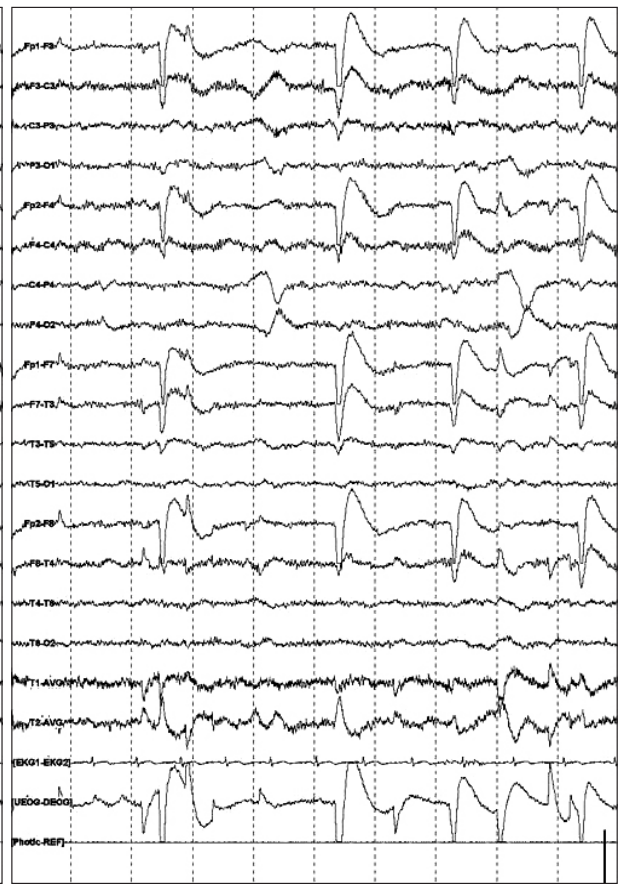

Fig. 1. The electroencephalography show slow wave before medication (zolpidem-off, A) and the reversal of slow wave to beta range fast activity after medication (zolpidem-on, B).
(A)
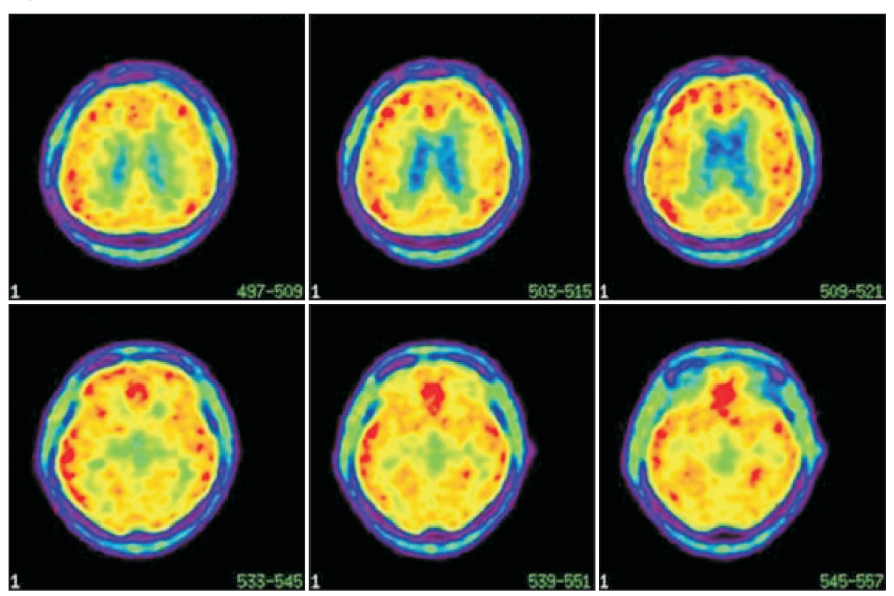

(B)
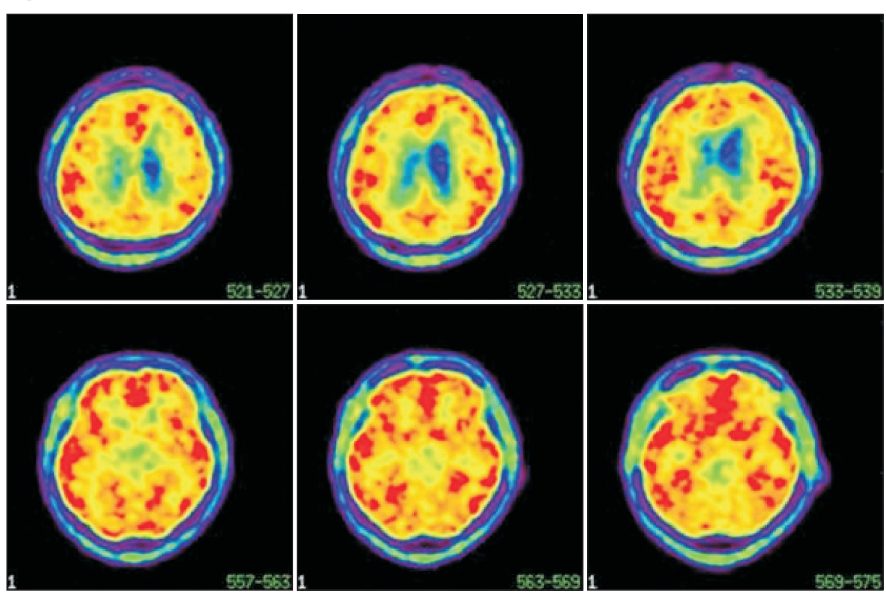

Fig. 2. F-18 flumazenil positron emission tomography $\left(\left[{ }^{18} \mathrm{~F}\right]\right.$-FMZ-PET $)$ show increased GABAergic receptor activity in bilateral frontoparietotemporal cortex at zolpidem-on (B), as compared to zolipdem-off (A).

lobe and improvement of cerebellar diaschisis on SPECT. $\left[{ }^{18} \mathrm{~F}\right]$-FMZ PET showed increased GABA receptor activity at zolpidem-on.

The effect of zolpidem on cerebral perfusion by SPECT was observed experimentally in animals and human $[2,7]$. The mechanism of zolpidem-induced increase in blood perfusion of injured brain is still unknown; but in an animal study, $\mathrm{GABA}_{\mathrm{A}}$ receptor modulated nitric oxide synthase activity in brain by administration of diazepam [8]. Zolpidem may have a similar effect and increase perfusion by increased nitric oxide concentration of brain, as it has affinity to the $\mathrm{GABA}_{\mathrm{A}}$ receptor as well.

Two cellular pathways could be initiated after brain injury, i.e., glutamate-utilizing and excitatory pathway [9] and GABA-induced neurodormant cells whose functions can be reversed by activation of modified GABA receptor 
(A)

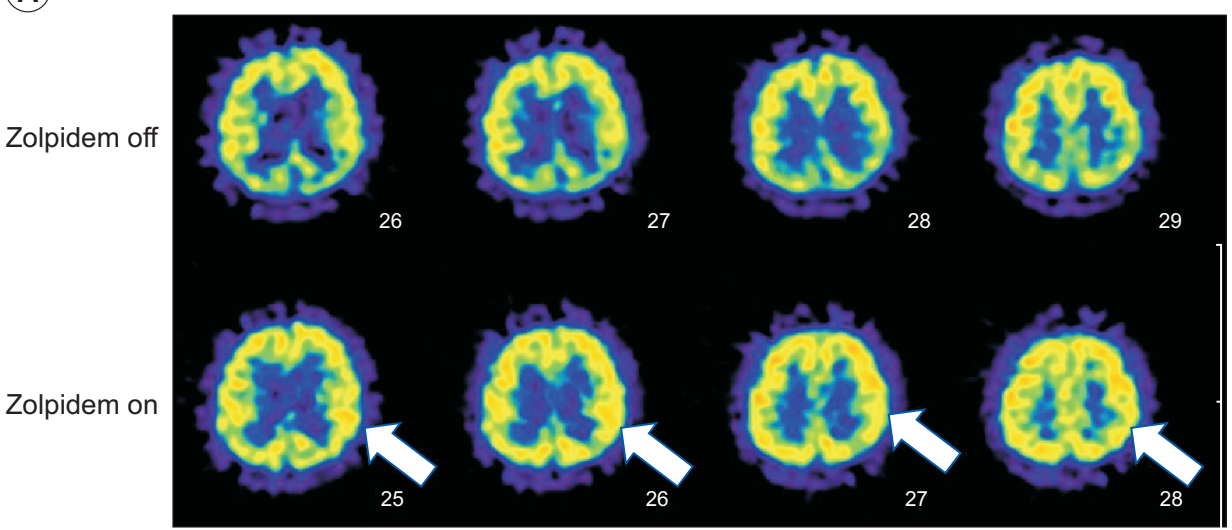

(B)

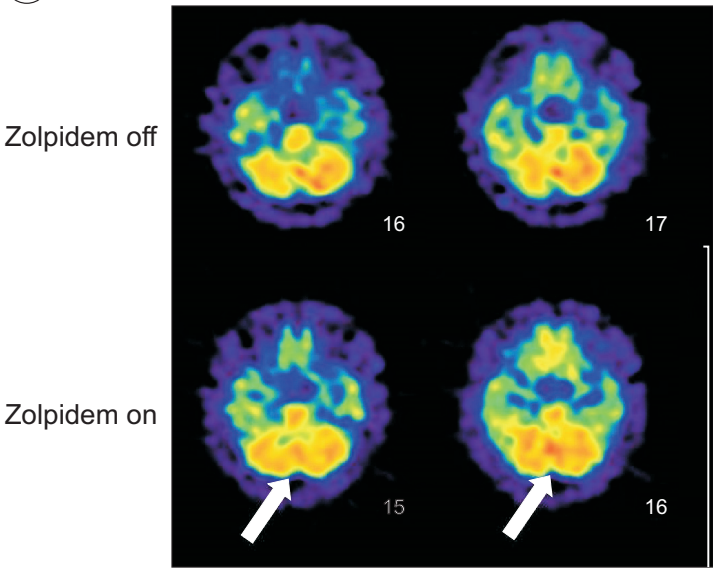

Fig. 3. Single photon emission computed tomography (SPECT) show increased perfusion of left parietal lobe after administration of zolpidem (A) and improvement of diaschisis in cerebellum (B).

[10]. Clauss and Nel [1] suggested that zolpidem binding to the modified GABA receptor that is supersensitized to inhibitory neurotransmitter after anoxic brain damage could cease abnormal cell metabolism. If dormant cells affect a large brain area, dramatic clinical changes could occur if these are switched-on by zolpidem. If only those who have large areas of dormant cells benefit from zolpidem, it may explain why all patients do not show improved cognition and behavior in large prospective studies $[3,4]$. In our patient, $\left[{ }^{18} \mathrm{~F}\right]$-FMZ-PET showed increased GABAergic receptor activity when zolpidem was administered, as compared to the zolpidem-off state. Flumazenil is a specific, reversibly binding high-affinity antagonist at the benzodiazepine site of the $\mathrm{GABA}_{\mathrm{A}}$ receptor by competitive inhibition of the omega receptors. In normal brain, decreased GABAergic receptor activity is expected when zolpidem and flumazenil are administered together. This was also confirmed by Clauss et al. [7] However, our case showed increased GABAergic receptor activity when zolpidem and flumazenil were adminis- tered. This may suggest that the zolpidem selectively acts on modified omega receptors after anoxic brain damage and consequently increases the affinity of down regulated GABA receptors.

Since none of the other GABAergic drugs showed this paradoxical arousal effect, it is likely that the high selectivity of zolpidem to the $\mathrm{GABA}_{\mathrm{A}}$ omega receptor alone and not the other 2 subtypes, mediates this effect. Highly selective GABA omega-1 receptor agonist, zolpidem leads to phosphorylation of the receptor and results in the termination of brain dormancy by exposing receptor-related protein that are targets for protein kinases [6]. This is the first case with evidence of zolpidem-induced altered affinity and function of GABA receptor by using $\left[{ }^{18} \mathrm{~F}\right]$-FMZPET in the human brain. However, the mechanism of zolpidem-induced modification of the omega receptor requires further research. 


\section{CONFLICT OF INTEREST}

No potential conflict of interest relevant to this article was reported.

\section{REFERENCES}

1. Clauss R, Nel W. Drug induced arousal from the permanent vegetative state. NeuroRehabilitation 2006;21:23-8.

2. Clauss RP, Guldenpfennig WM, Nel HW, Sathekge MM, Venkannagari RR. Extraordinary arousal from semi-comatose state on zolpidem: a case report. S Afr Med J 2000;90:68-72.

3. Whyte J, Rajan R, Rosenbaum A, Katz D, Kalmar K, Seel R, et al. Zolpidem and restoration of consciousness. Am J Phys Med Rehabil 2014;93:101-13.

4. Thonnard M, Gosseries O, Demertzi A, Lugo Z, Vanhaudenhuyse A, Bruno MA, et al. Effect of zolpidem in chronic disorders of consciousness: a prospective open-label study. Funct Neurol 2013;28:259-64.

5. Brefel-Courbon C, Payoux P, Ory F, Sommet A, Slaoui
T, Raboyeau G, et al. Clinical and imaging evidence of zolpidem effect in hypoxic encephalopathy. Ann Neurol 2007;62:102-5.

6. Du B, Shan A, Zhang Y, Zhong X, Chen D, Cai K. Zolpidem arouses patients in vegetative state after brain injury: quantitative evaluation and indications. Am J Med Sci 2014;347:178-82.

7. Clauss RP, Dormehl IC, Kilian E, Louw WK, Nel WH, Oliver DW. Cerebral blood perfusion after treatment with zolpidem and flumazenil in the baboon. Arzneimittelforschung 2002;52:740-4.

8. Paul V, Subramanian EH, Rajasekaran K. Pharmacological evidence for a role of gamma-aminobutyric acid A receptor mechanism in modulating nitric oxide synthase activity in rat brain. Neurochem Int 2001;38:209-11.

9. Nicotera P, Orrenius S. The role of calcium in apoptosis. Cell Calcium 1998;23:173-80.

10. Clauss RP, Nel WH. Effect of zolpidem on brain injury and diaschisis as detected by $99 \mathrm{mTc}$ HMPAO brain SPECT in humans. Arzneimittelforschung 2004;54:641-6. 